\title{
Geech: onomatopeya y cognición en el quechua central
}

\author{
"Geech": onomatopoeia and cognition in Central Quechua \\ Rolando Rocha Martínez \\ Universidad Nacional Mayor de San Marcos, Lima, Perú \\ Contacto: rrocham@unmsm.edu.pe \\ https://orcid.org/0000-0002-9162-9861
}

\begin{abstract}
Resumen
Se analiza un conjunto de formas onomatopéyicas ocurrentes en el quechua central, las cuales, contrariamente a la consideración estructural tradicional, evidencian una organización sistemática consistente que se explicita en mecanismos rastreables de conformación onomatopéyica como la reduplicación y la cantidad vocálica. En tal sentido, el objetivo principal es realizar un trabajo de recopilación de data real a fin de evaluar el comportamiento de la conformación de onomatopeyas a través de la medición de los correlatos acústicos, por ejemplo, el de cantidad. Se detectan configuraciones conceptuales en las cuales ciertas secuencias de vocales y consonantes se vinculan con un marco conceptual que expresa la forma en que los hablantes de quechua categorizan las emisiones animales. Sobre este último aspecto conviene afirmar que no se trata de repeticiones o imitaciones de los animales, sino de formas en las cuales la asociación sonido y significado se expresa en patrones fonológico-conceptuales específicos.
\end{abstract}

Palabras clave: Onomatopeya; Quechua central; Esquema fonológicoconceptual; Onomátopo.

\begin{abstract}
A set of onomatopoeic forms occurring in central Quechua are analyzed, which, contrary to the traditional structural consideration, show a consistent systematic organization, which is explained by traceable mechanisms of onomatopoeic conformation such as reduplication and vowel quantity. In this context, the main objective is to develop a job of real data collection in order to evaluate the behavior of the conformation of the onomatopoeias through the measurement of acoustic correlates, such as quantity. Conceptual configurations are detected, in which certain vowel and consonant sequences are linked to a conceptual framework that expresses the way in which Quechua speakers categorize animal emissions. In this last aspect, it should be stated that it is not a matter of repetitions or imitations of animals, but of categorizations in which the association between sound and meaning is expressed in specific phonological-conceptual patterns.
\end{abstract}

Keywords: Onomatopoeia; Central Quechua; Phonological-conceptual Scheme, Onomatopo 


\section{Introducción}

Las onomatopeyas no siempre fueron consideradas como un objeto de estudio consistente con el objetivo de describir lo sistemático que, en el ámbito de la tradición lingüística de corte estructural, caracterizaba a la estructura lingüística; es decir, se consideraba que las formas onomatopéyicas carecían de sistematicidad. La asunción de la lengua como organización estructurada de oposiciones las excluía, debido al estatus teórico del signo lingüístico como unidad arbitraria, arbitrariedad que no era detectable en las motivaciones evidentes de los recursos onomatopéyicos en las lenguas naturales. Así, tal y como se plantea en las reflexiones saussureanas, “[...] las onomatopeyas nunca son elementos orgánicos de un sistema lingüístico" (De Saussure, 1945, p. 95). De acuerdo con el aserto anterior, el objeto de estudio de la lingüística se ciñe a lo regular y sistemático: la onomatopeya, como contraparte, está fuera de la discusión y, en consecuencia, su abordaje empírico fue considerado irrelevante.

A pesar de este inicio infructuoso, la ocurrencia de onomatopeyas en las lenguas del mundo es patente, y la característica sine qua non de estas formas lingüísticas es la evidente motivación que subyace a su manifestación concreta; es decir, se trata de formas que configuran lingüísticamente diversos tipos de emisiones del mundo natural (ya sea de entidades animadas o inanimadas). En tanto que tal, resulta razonable considerar que, en las formas onomatopéyicas, la arbitrariedad del signo queda descartada. Por otro lado, muchas de las formas registradas evidencian similitudes inquietantes interlingüísticamente; sin embargo, es menester aclarar que no se trata de una imitación de la realidad acústica que intenta expresar, sino de una interpretación del ruido (la emisión animal, entre otros potenciales sonidos), en términos lingüísticos. Por consiguiente, la fonología cumple un papel importante en esta categorización de la realidad acústica no lingüística, a fin de conformar expresiones con unidades discretas definidas y mecanismos de formación de onomatopeyas específicos como la reduplicación y el alargamiento.

Con base en estas consideraciones previas, nuestro estudio ${ }^{1}$ pretende determinar los mecanismos que orientan la configuración de las onomatopeyas en el quechua central. Esta tarea puede dividirse en la delimitación de los 
mecanismos prototípicos que los hablantes del quechua central utilizan para crear formas de este tipo, por un lado, y la discusión sobre la potencial continuidad entre lo lingüístico y extralingüístico en el quechua, por otro. Ambos objetivos se orientarán a la clarificación acerca del uso de categorías fonológicas con correlatos semánticos que se manifiestan en el discurso y cuya motivación es pasible de detección en el entorno inmediato de los quechuahablantes, quienes verbalizan las onomatopeyas dirigidas por la experiencia y apropiación del entorno en el que se desenvuelven a diario. Un concepto útil para establecer generalizaciones aceptables sobre las onomatopeyas en el quechua central es el de onomátopo, planteado por Moreno Cabrera (2016).

\section{Justificación}

Nuestro trabajo ha consistido en la recopilación de evidencia empírica en audios de alta calidad, con la finalidad de proyectar un estudio de largo alcance sobre la forma en que se explicitan las onomatopeyas en los distintos dialectos quechuas. De esta manera, consideramos que es importante contar con un registro detallado de datos que puedan servir en la constitución de repositorios virtuales que sean de utilidad en términos aplicativos. Por ello, consideramos que nuestro estudio se enmarca en los procedimientos de documentación lingüística. Los trabajos de este tipo han adquirido relevancia a escala mundial y se justifican por diversas razones. En primer lugar, la vitalidad de las minorías lingüísticas se encuentra en serio riesgo, y las lenguas peruanas no son la excepción.

La diversidad lingüística merece el reconocimiento de su importancia y los trabajos de documentación se erigen como procedimientos necesarios, dado que permiten la obtención de muestras heterogéneas de las lenguas vivas y los diversos fenómenos que se derivan de ellas (Himmelmann, 1998). El trabajo de documentación, visto en esos términos, y dado el espectro amplio y heterogéneo de datos que aborda, recibe el influjo de diversas subdisciplinas de la lingüística: el análisis del discurso, la adquisición del lenguaje, la fonética, la lingüística teórica, entre otras (Himmelmann, 1998, p. 167). A propósito de lo heteróclito de su campo de acción, la documentación lingüística se apropia de las nuevas tecnologías para realizar un trabajo profundo y confiable de registro de corpus lingüístico cuya finalidad, más allá de la descripción de datos, consiste en la 
preservación y la accesibilidad de las lenguas en peligro de extinción (Kritikos, 2015 , p. 243). Este objetivo es apremiante para efectos de la visibilidad y la permanencia de las lenguas cuyo nivel de resiliencia y vitalidad es bajo, pues hace posible, en términos prácticos, que la comunidad de hablantes se comprometa con la acción de fortalecer la lengua en peligro.

Es esta la razón por la cual la documentación de lenguas presenta un componente ético que es fundamental para asegurar el derecho de propiedad de quienes actualizan la lengua en situaciones reales. Este trabajo, que supera las prácticas tradicionales de recopilación del corpus lingüístico con herramientas que se limitan a la versión de los datos propuesta por quien analizó la lengua, permitirá contar con datos directos a fin de que, posteriormente, sea posible tener un registro abierto y accesible que permita una mejor elicitación y análisis de la lengua en rigor. Por lo anterior, el presente estudio se justifica en el marco de las políticas lingüísticas de reconocimiento y fortalecimiento de las minorías lingüísticas de nuestro país mediante la incorporación tecnológica y la necesidad de permitir la accesibilidad de los datos, pues hará posible trabajar y contar con muestras reales, pasibles de análisis y codificación (Sadick y Nartey, 2014, p. 79). Este trabajo de análisis fonético-fonológico, además de permitir que la lengua cuente con un pequeño repositorio de la lengua viva, aportará en lo concerniente a la implementación de una metodología diferente para acceder y analizar el corpus, además de suponer un aporte en la detección precisa de los sonidos de la lengua. Dado el carácter multifuncional del trabajo de documentación, es posible que los datos y el análisis se usen para realizar trabajos de alfabetización en la lengua indígena, que partan de la medición técnica de la muestra a través del planteamiento de los correlatos fonético-fonológicos específicos, de manera que la subjetividad de la audición directa de las grabaciones obtenidas sea desplazada por un sistema de medición acorde con el desarrollo científico-tecnológico. Una de las ventajas del PRAAT es que permite trabajar con audios en formato wav, es decir, una versión no comprimida de la muestra acústica.

\section{Planteamiento del problema}

En el quechua central se verbalizan elementos onomatopéyicos asociados con diversos referentes acústicos que se corresponden con la experiencia vital del 
quechuahablante (emisiones animales, ruidos de objetos o máquinas, entre otros). Las formas en que las onomatopeyas se constituyen son de diverso tipo: reduplicación parcial y reduplicación total, alargamiento de vocal e, incluso, la interacción entre estos. Los datos, al parecer heteróclitos, podrían erigirse en evidencia sobre posibles mecanismos que el hablante de quechua prefiere. Además, las onomatopeyas en el quechua central (aunque no es privativo de esta lengua), al configurarse con base en la experiencia acústica a la que está expuesta el hablante, presentan una motivación sólida, de manera que es razonable realizar una sistematización de las expresiones onomatopéyicas y dilucidar los mecanismos cognitivos involucrados. Algunos casos de la muestra son presentados en (1) a continuación:
(1) chap chap 'sonido que hace el chancho al comer' chiwii chiwii 'el canto del chiwi (ave similar al zorzal pero negra)'
gaw gaw 'el sonido producido por el estómago vacío' geech 'el sonido producido cuando se abre una puerta'
han han 'el ladrido del perro' haachin haachin 'el rebuzno del burro' kar kar 'ruido que hacen los loros en grupo' lap lap 'el sonido que produce el perro al lamer' тии 'el sonido que produce la vaca' maa 'el sonido que produce la oveja' palchacha palchacha paq paq 'el sonido de la cancha cuando revienta' par par 'ruido que hace al volar' pichiw pichiw 'el canto del gorrión' plachaqq 'el sonido de una bofetada fuerte' sap sap 'el sonido que produce un ave al volar' taq taq 'el sonido de la puerta cuando alguien toca' 


$\begin{array}{ll}\text { taqraqq } & \text { 'el sonido producido cuando cae un objeto' } \\ \text { titii } & \text { 'el sonido del claxon del carro' } \\ \text { tukuu tukuu } & \text { 'el sonido que produce la lechuza' } \\ \text { wishik wishik } & \text { 'el sonido que produce el cuy' } \\ \text { yuk yuk } & \text { 'el canto del yukish (zorzal)' } \\ \text { zap zap } & \text { 'ruido de las alas del zorzal' }\end{array}$

Los datos en (1) refieren realidades que, de forma robusta, se relacionan con las emisiones animales, pero no es el único referente. Como se aprecia, es posible que las onomatopeyas se vinculen con el ruido de objetos, de partes del cuerpo humano, entre otros. A la luz de la evidencia empírica, es pertinente formular las siguientes preguntas:

a) ¿Qué mecanismos fonológico-conceptuales están involucrados en la constitución de onomatopeyas en el quechua central?

b) ¿Cuál de estos mecanismos es prototípico en el quechua central?

c) ¿Qué motivaciones subyacen a la ocurrencia de onomatopeyas en el quechua central?

\section{Objetivos}

Los objetivos que pretendemos alcanzar con este estudio son los siguientes:

\subsection{Objetivo general}

Precisar los mecanismos fonológico-cognitivos que determinan la conformación de onomatopeyas en el quechua central

\subsection{Objetivos especificos}

a) Definir los correlatos acústicos de los mecanismos fonológicos involucrados en la conformación de onomatopeyas en el quechua central.

b) Documentar acústicamente la forma en que se explicitan las onomatopeyas y determinar las motivaciones involucradas en su constitución.

c) Establecer cuáles de los diversos mecanismos fonológico-conceptuales es el más prototípico para el hablante de quechua. 


\section{Hipótesis}

En el quechua central se presentan onomatopeyas motivadas por el marco experiencial de los quechuahablantes, cuyos mecanismos presentan una jerarquía de prototipicidad reflejada en la mayor o menor ocurrencia.

\section{Metodología}

El estudio es de tipo experimental, pues pretende ofrecer una explicación razonable de la ocurrencia de mecanismos de formación de onomatopeyas en quechua central, con la consiguiente documentación de los correlatos acústicos si es que los hubiere. Este trabajo se realizará a través de distintos pasos para asegurar una documentación razonable de datos acústicos en la lengua quechua. En virtud de que se trata de un trabajo de documentación fonética, cuyos resultados permitirán formular conjeturas razonables en el nivel fonológico, la metodología asumida se enmarca en el ámbito de la lingüística documental; de esta manera, se usarán instrumentos de recopilación (esta labor directamente enmarcada en el trabajo de documentación) y medición (Nartey, 2013, p. 1), que permitan superar los escollos de procedimientos ortodoxos en el ámbito del trabajo de campo.

Entre los problemas derivados de la labor del investigador, es posible señalar los siguientes (Nathan, 2010, p. 262): a) la selección de equipos cuyos audios eran defectuosos o de baja calidad, b) una metodología en la que las grabaciones se realizaban con los hablantes nativos demasiado lejos o los micrófonos mal direccionados, y c) un producto del recojo poco atractivo para oír y para usar en la enseñanza de la lengua. Los problemas del trabajo de campo tradicional serán superados mediante la escogencia de herramientas adecuadas, tanto para el recojo de datos como para el análisis de estos con la finalidad de obtener un trabajo de documentación real, que haga posible acceder a los datos en cualquier momento y para cumplir cualquier objetivo.

En virtud de que el investigador ostenta la capacidad para obtener y utilizar equipos adecuados, además de participar de la experiencia de acceder directamente a la lengua natural, se deriva de este aspecto una dimensión ética por parte del lingüista, pues debe asumir como objetivo el registro de datos con un alto estándar de calidad (Nathan, 2010, p. 265). El trabajo de documentación 
presupone la interacción sinérgica con los nativohablantes, pues estos son conscientes del repliegue de la lengua en las comunidades, de manera que ellos podrían contribuir de forma activa en el trabajo per se; a fin de que esta labor sea posible, es necesario que el investigador y los hablantes de la lengua lleguen a un trabajo consensuado que beneficie y permita un registro meticuloso en el que las opiniones de los usuarios activos de la lengua sean consideradas y realzadas, pues de esa forma será viable la labor propuesta por la lingüística documental (Riemann, 2010, p. 255). El análisis, de forma consistente con el trabajo de documentación y la pesquisa fonológica que se pretende realizar, estará conformado por una serie de fases que incluirá la preparación de la herramienta de recolección de datos, la labor misma de documentación y el análisis fonético mediante el programa informático Praat. En cuanto a las etapas que conformarán el estudio, estás son las siguientes:

\subsection{Elaboración del estado del arte sobre las onomatopeyas en quechua}

Esta actividad fue medular para reconocer el repertorio de mecanismos de formación onomatopéyica y la muestra recogida por los diversos investigadores. Es importante realizar un rastreo de la interpretación que se ha efectuado de los datos recolectados, las técnicas de recopilación del corpus y las conjeturas devenidas del análisis de este. Sobre el particular, se consultó el inventario de onomatopeyas, específicamente en la variedad de Apurímac (Sierra Peña, 2010).

6.2. Organización del instrumento para el trabajo de documentación de onomatopeyas en quechua central

El cuestionario se organizará a partir de la consulta de diccionarios o fuentes lexicográficas diversas. Las onomatopeyas serán situadas en un contexto específico, con la finalidad de contar con evidencia empírica en enunciados completos y no en entradas aisladas.

\subsection{Documentación de datos de onomatopeyas en quechua central}

Una vez estructurado el cuestionario, se dispondrá a realizar sesiones de grabación. Para ello es necesario el uso de una grabadora portátil de 4 pistas a estado sólido marca Tascam, modelo DR-40. Esta hace posible la obtención de evidencia acústica en formato wav (no comprimido). Por otro lado, es posible obtener 
muestras con el menor nivel de interferencias mediante el uso de un micrófono phantom marca Shure, modelo WH30, cuyo patrón de captación de cardioide posibilita el aislamiento de las fuentes sonoras no deseadas y la obtención de audios de muy buena calidad.

\subsection{Análisis acústico de datos mediante el Praat}

Para realizar un análisis acústico acorde con la incorporación de tecnología que permita superar la intuición derivada de la audición directa, se utilizará el programa informático Praat, software creado en 1992 por Paul Boersma y David Weenink (1999). Esta herramienta presenta un diseño que hace posible la obtención de datos para realizar mediciones acústicas de alta calidad, además de presentaciones de gráficos con las mismas características. El programa es funcional en varios sentidos: se ajusta a diversas plataformas (Windows, Linux y Mac) y es de acceso libre. Los creadores realizan un mantenimiento permanente del mismo.

6.5. Elaboración de la propuesta fonológica sobre los mecanismos fonológicocognitivos involucrados en la constitución de onomatopeyas

Para elaborar hipótesis razonables acerca de los mecanismos fonológicos involucrados en la configuración de formas onomatopéyicas, se usarán los presupuestos teóricos de la lingüística cognitiva; es decir, desde una óptica que, a diferencia de otros trabajos de descripción gramatical específicos sobre el quechua (Rocha, 2010; Rocha, 2011), vinculan los aspectos gramaticales con lo extralingüístico.

\section{Marco teórico}

La propuesta teórica que asumiremos es la LC (Lingüística Cognitiva). En rigor, la teoría cognitiva considera que el lenguaje forma un continuo con la cognición en general, de manera que hace posible la construcción de significados. En tal sentido, el lenguaje emerge y no se trataría de un dispositivo especializado y autónomo. Visto de esa forma, la propuesta cognitiva se decanta por la asunción de que el lenguaje permite construir significados, de manera que constituye una propuesta basada en el uso. En términos metodológicos, los diversos autores cognitivistas no necesariamente prescinden de la idealización de los datos; sin embar- 
go, nuestra propuesta se orienta por la recolección de una muestra acústica real, a fin de analizar la data de forma directa y evaluar su comportamiento. Por otro lado, en el marco de esta propuesta, es menester considerar variables diferentes a las de los enfoques formalistas, puesto que se vinculan los datos con la cognición general, lo cual supone que el marco experiencial es fundamental para la internalización de esquemas semánticos.

En suma, de acuerdo con los presupuestos de la LC sobre el lenguaje y su funcionamiento, este depende de la cognición, de forma que se vincula con la percepción, las emociones, el razonamiento, la memoria, entre otras habilidades cognitivas. La consideración de que estos procesos están integrados en el proceso cognitivo implica conferirle un estatus mental (Taylor, 2003, p. 5). Si bien es cierto existe una contraposición de fondo entre el generativismo y las propuestas de tipo cognitivo, lo que los cognitivistas impugnan es que el carácter innato suponga que el lenguaje es autónomo de la cognición general, no que existan condiciones innatas para el lenguaje (Croft \& Cruse, 2004, p. 2). En cuanto al estatus del lenguaje en el marco de la cognición general, Goldberg propone que el conocimiento de tipo lingüístico es consistente con el conocimiento mismo (1996, p. 3). En cuanto a la función medular del lenguaje, también se asume, como ya se dijo, que esta consiste en "[...] expresar significado. Una gramática debería por tanto mostrar de la forma más directa posible cómo los parámetros de forma se unen con parámetros de significado" (Lakoff, 1987, p. 583). En cuanto a las onomatopeyas, estas permiten explicitar la forma en que los sonidos se conceptualizan a partir de la experiencia. Ello supone que el hablante no intenta imitar, sería absurdo que intente replicar el ruido generado por las alas de un ave. Lo que es razonable en este caso es que los hablantes se permiten categorizar esta experiencia y la vinculan con categorías de significado que son predecibles. En tanto que tal, disentimos de la propuesta inicial de De Saussure (1945, p. 95), quien considera que las onomatopeyas carecen de organicidad o son elementos marginales del sistema lingüístico. Consideramos que las onomatopeyas en quechua son el producto de claros esquemas mentales que permiten establecer la asociación entre el sonido y el significado que intenta explicitarse a través de estas formas. Por ello es pertinente la siguiente afirmación: 
Aunque es cierto que las onomatopeyas son creaciones propias de cada lengua, y por lo tanto, con unos rasgos únicos para cada idioma, no es menos cierto el hecho de que puedan considerarse una 'categoría universal' con unas características estructurales, semánticas y pragmáticas comunes a las lenguas del mundo (Voeltz y Kilian-Hatz 1999). (Ibarretxe, 2012)

Si bien es cierto, la asociación se establece entre emisiones de tipo animal y la fonología de la lengua, el espectro es mucho más amplio, dado que es posible detectar onomatopeyas vinculadas con los correlatos acústicos reales que derivan de la forma en que aletean las aves o la manera en que los objetos impactan con superficies duras. En consecuencia, será menester recurrir a un concepto que posibilite definir las categorías involucradas en la configuración de esquemas cognitivos específicos en el quechua, aunque estos sean extrapolables a otras lenguas. De lo anterior se sigue que los procedimientos onomatopéyicos, corroborables en las lenguas naturales, “[...] son el resultado de la interpretación lingüística de sonidos naturales. Esa interpretación está guiada por principios generales fonosimbólicos y se puede realizar en las dos articulaciones del lenguaje" (Moreno Cabrera, 2016).

La constitución de patrones regulares es detectable interlingüísticamente. Esto quiere decir que no es privativo de una lengua en concreto, aunque podrían existir ciertas divergencias. Así, el sonido emitido por los pájaros y por los pollos es agudo y suele ser expresado en diversas lenguas mediante una sílaba que contiene una consonante oclusiva o fricativa cuyo núcleo es la vocal /i/. Moreno Cabrera (2016, p. 135) presenta datos como los de la familia romance, que comprende lenguas como el latín: pipio; el español, el portugués: piar; el catalán: piular, el italiano: pigolare; y el francés: piauler. También plantea datos de la familia eslava: ruso: чирикать [chirikat']; búlgaro: пискам [piskam]; serbio: пиштати [pishtati]; polaco: piszczeć. No son los únicos datos que establece, pero tomamos los que genéticamente se alejan más, de acuerdo con los criterios tipológicos para asumir una muestra significativa (Hengeveld, 2003, p. 89). A fin de brindar una primera muestra, presentamos las onomatopeyas siguientes: 


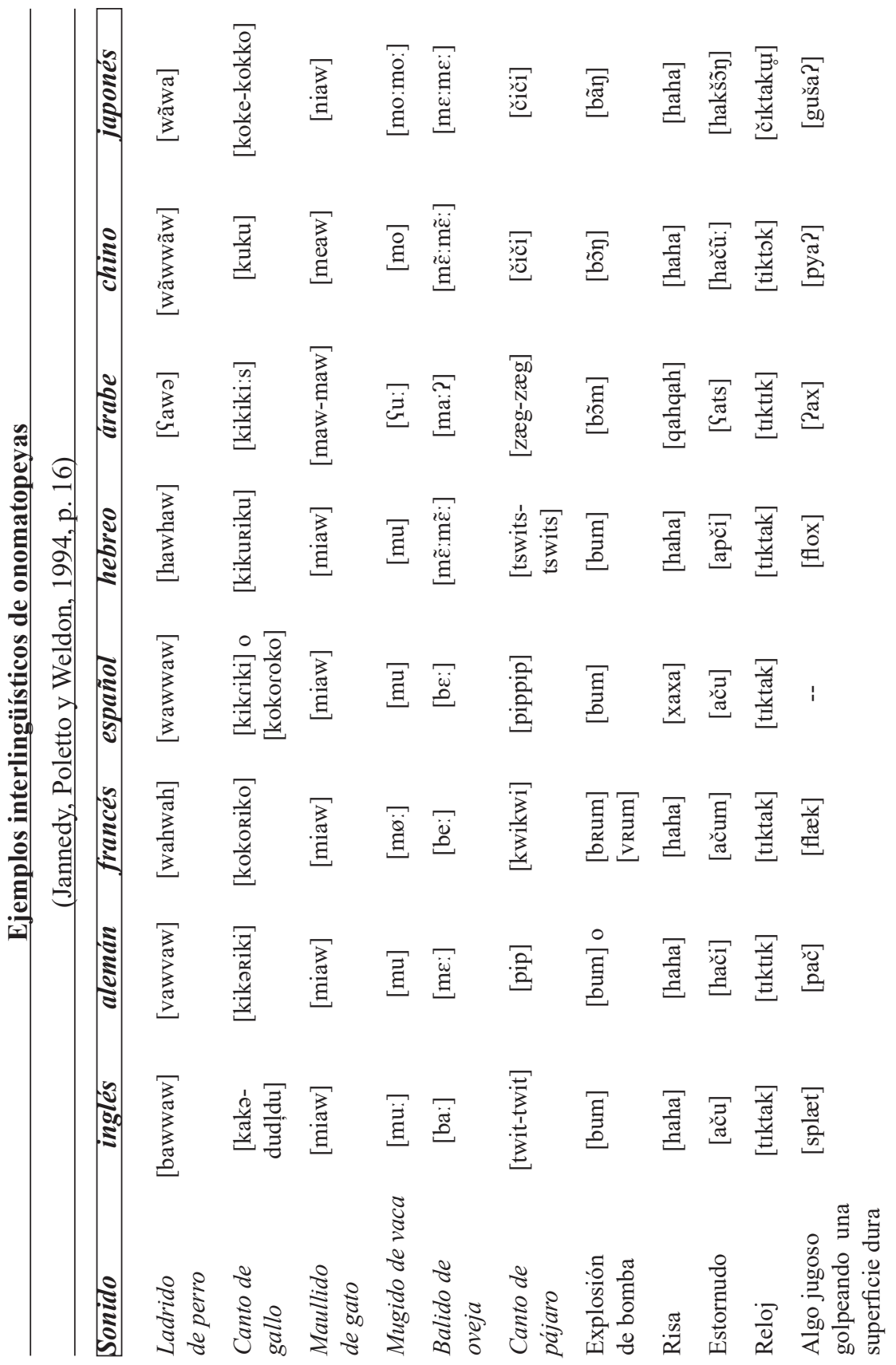


Notamos que existen coincidencias consistentes en las lenguas recopiladas por los autores, sobre todo en correlatos de la realidad que no implican una complejidad del ruido que se categoriza fonológicamente y cuya réplica fonética se explicita. Por esa razón, Moreno Cabrera, siguiendo a Voronin (2005), asume el concepto de onomátopo. Los onomátopos son "[...] raíces con un significado icónico que dan lugar a diversas onomatopeyas en diversas lenguas" (Moreno Cabrera, 2016, p. 136). Los onomátopos están motivados, de forma que dista de la arbitrariedad clásica que se le confiere al signo lingüístico. Un caso concreto de onomátopo es la forma pi o pip, para referirse al sonido del pito o al sonido de los pájaros. Esta estructura es reconocible interlingüísticamente y es robusta por su regularidad y por incluir los mismos sonidos.

Nuestro propósito a continuación será evaluar la evidencia empírica recogida. Usaremos la noción de onomátopo y definiremos las implicancias semánticas de esta forma de reconfigurar la realidad de manera fonológica.

\section{Análisis de datos}

En primer lugar, detallaremos cuáles son los mecanismos usuales en el quechua para estructurar onomatopeyas. De acuerdo con los datos recopilados, tanto la reduplicación (ya sea total o parcial) como el alargamiento son los mecanismos usados para estructurar onomatopeyas. Algunos de los datos son los siguientes:

(1) Reduplicación total o parcial
chap chap
'sonido que hace el chancho al comer'
wishik wishik
'el sonido que produce el cuy'
pichiw pichiw
'el canto del gorrión'

$\begin{array}{ll}\text { titii } & \text { 'el sonido del claxon del carro' } \\ \text { tukuи tukuu } & \text { 'el sonido que produce la lechuza' } \\ \text { plachaqq } & \text { 'el sonido de una bofetada fuerte' } \\ \text { aqraqq } & \text { 'el sonido producido cuando cae un objeto' }\end{array}$

(2) V: o CC

En los datos de (1), las bases léxicas chap, wishik y pichiw se reduplican para dar lugar a las formas onomatopéyicas vinculadas con emisiones animales. Es importante indicar que la evidencia empírica es consistente con la asociación entre 
el mecanismo de reduplicación y el simbolismo, aunque en lenguas europeas este recurso es menos frecuente (Hinton, Nichols \& Ohala, 1994, p. 9). Sin embargo, no en todos los casos son producciones animales las que se conceptualizan en quechua, ya que chap se asocia con el ruido que hace el chancho al comer. Por otro lado, el mecanismo adicional que usan los quechuahablantes al estructurar onomatopeyas es el alargamiento de vocales y de consonantes. Estos dos patrones presentan implicancias silábicas, dado que las consonantes que aumentan su tiempo de duración se posicionan a final de sílaba. En el caso de las vocales, estas solo se manifiestan con cantidad en casos en los que las onomatopeyas categorizan ruidos con duración. Esto quiere decir que la experiencia condiciona la ocurrencia y el uso de los mecanismos lingüísticos de cantidad. En atención a lo dicho, se requiere en este caso concreto la evidencia de la duración y, por consiguiente, los correlatos fonéticos que permitan corroborar tales patrones lingüísticos. En rigor, hemos detectado, en efecto, una mayor duración en vocales. Este rasgo es consistente con la detección de onomatopeyas cuyo correlato son sonidos con mayor tiempo de duración; por ejemplo, el canto de un ave. Los golpes, no obstante, requieren vocales cortas.
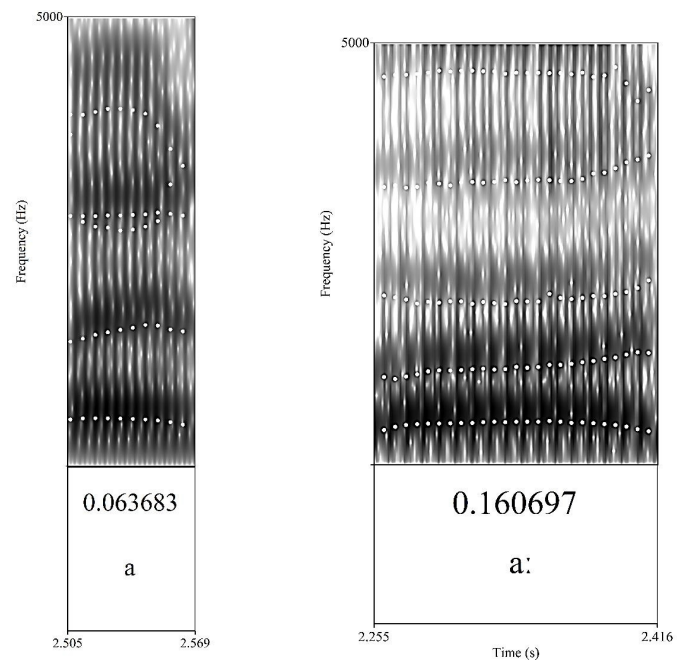

Figura 1. Espectrograma de vocal corta [a] en la onomatopeya [tahtah] 'ruido que genera la acción de tocar la puerta'y espectrograma de vocal larga [a:] en la onomatopeya [ma:] 'emisión de la oveja' en quechua. 

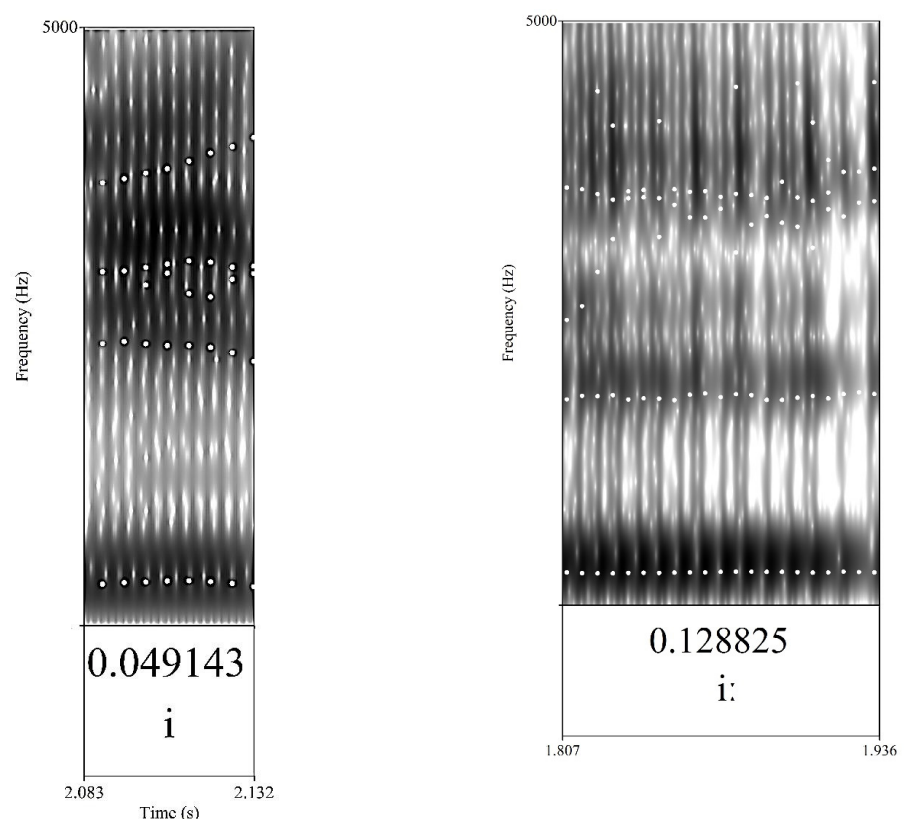

Figura 2. Espectrograma de vocal corta [i] y la vocal larga [i:] en la onomatopeya [titi:] 'emisión del claxon de un carro'.

En las imágenes anteriores notamos duraciones diferentes para las vocales cortas: 0,063 milisegundos para la vocal central baja abierta no redondeada [a] y 0,049 milisegundos en el caso de la vocal alta anterior [i]. Sus correspondientes correlatos con mayor duración son la vocal central baja larga [a:], cuya duración es de 0,160 milisegundos, y la vocal alta anterior larga [i:], de 0,128 milisegundos. La duración, en rigor, es el correlato usado para configurar fonológicamente sonidos con mayor o menor extensión en la realidad.

En el caso del sonido que se representa con la grafía $<\mathrm{q}>$, se trata de un sonido glotal $[\mathrm{h}]$. La duración de este también varía en las onomatopeyas que lo incluyen. Si bien siempre se posiciona a final de palabra o de sílaba, varía si el sonido que representa fonosimbólicamente es más prolongado o no. Nuevamente, el marco experiencial juega un rol importante en la forma en que se categoriza el sonido. Así, el siguiente espectrograma será ilustrativo al respecto. 

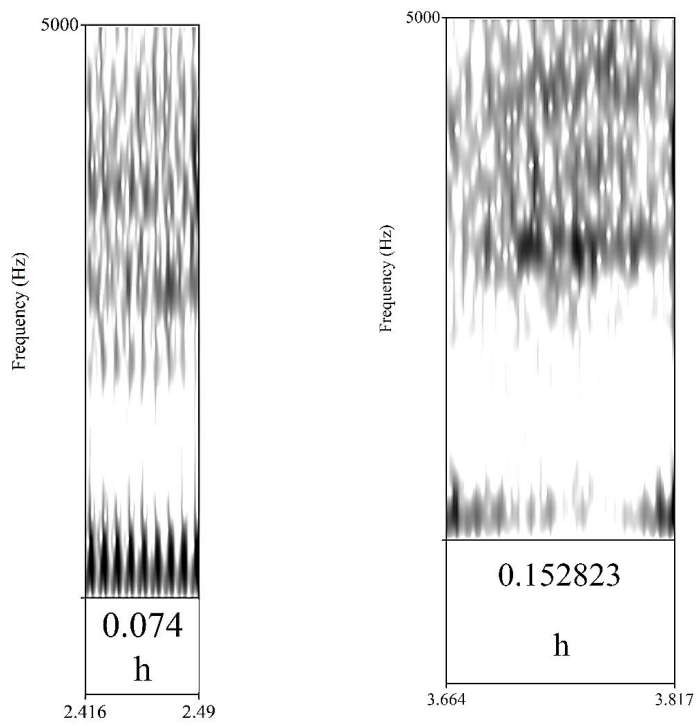

Figura 3. Sonidos fricativos glotales [h] en la onomatopeya [pahpah] 'sonido de la cancha cuando revienta' y en la onomatopeya [platfah:] 'sonido de una bofetada fuerte'.

La diferencia de duración es de 0,074 milisegundos en la onomatopeya vinculada con el ruido que genera la cancha al reventar (que es un sonido de menor duración) y de 0,152 en el caso de la onomatopeya asociada con la bofetada fuerte. La intensidad de este último se configura a través de una mayor duración del sonido glotal.

Si bien estos correlatos fonéticos son consistentes con la manifestación de onomatopeyas, requerimos de un análisis acerca de cómo se conformarían estos patrones acústicos en correlación con esquemas de sonidos. En tal sentido, consideramos que la evidencia acústica presentada haría posible el planteamiento de mecanismos fonosimbólicos. Dado que hemos requerido de la noción de onomátopo, nuestro siguiente paso será determinar cuáles son los que se vinculan con las onomatopeyas en el quechua central.

El primer onomátopo que proponemos está configurado con vocales anteriores, las cuales presentan asociación con un valor del sonido conceptualizado: 
el carácter agudo de este. Recordemos que Moreno Cabrera (2016) plantea el onomátopo pi para las onomatopeyas del pitido o la emisión de las aves. Sostenemos que, en el caso del quechua central, este se asocia con cualquier sonido categorizado como agudo. A continuación el onomátopo en cuestión.

$$
\mathrm{V}^{\mathrm{ANT}}+\mathrm{OBST}^{\mathrm{COR}}+\left(\mathrm{V}^{\mathrm{ANT}}\right)
$$

El onomátopo anterior representa la configuración de onomatopeyas que representan sonidos agudos. Tal es el caso de las onomatopeyas wishik wishik 'el sonido que produce el cuy', titii 'el sonido del claxon del carro' y la onomatopeya que encabeza el título de nuestro estudio, a saber, geech 'el sonido producido cuando se abre la puerta', entre otros. Otro onomátopo que representa más bien la conceptualización de sonidos graves es el siguiente:

$$
\mathrm{V}^{\mathrm{abierta}}+\mathrm{OBST}[\mathrm{OBST}]+\mathrm{V}^{\mathrm{abierta}}
$$

El onomátopo anterior se vincula con la siguiente parcela de datos. Cada uno de estos se corresponde con sonidos graves que se categorizan mediante la secuencia propuesta. Para facilitar la comprensión de las onomatopeyas, estas se proponen a continuación en (3).

(3) paq paq 'el sonido de la cancha cuando revienta' plachaqq 'el sonido de una bofetada fuerte' taq taq 'el sonido de la puerta cuando alguien toca' taqraqq 'el sonido producido cuando cae un objeto'

Dado que el onomátopo no es simplemente una abstracción que opera al margen del significado, planteamos la relación consistente entre el marco experiencial, los rasgos semánticos y el onomátopo en cuestión. Esta relación incluye la secuencia de sonidos que se vinculan con los significados derivados de las vivencias del hablante de quechua. Aunque estos datos se corresponden con el quechua central, es posible predecir que los diversos dialectos quechuas presentan mecanismos afines. 

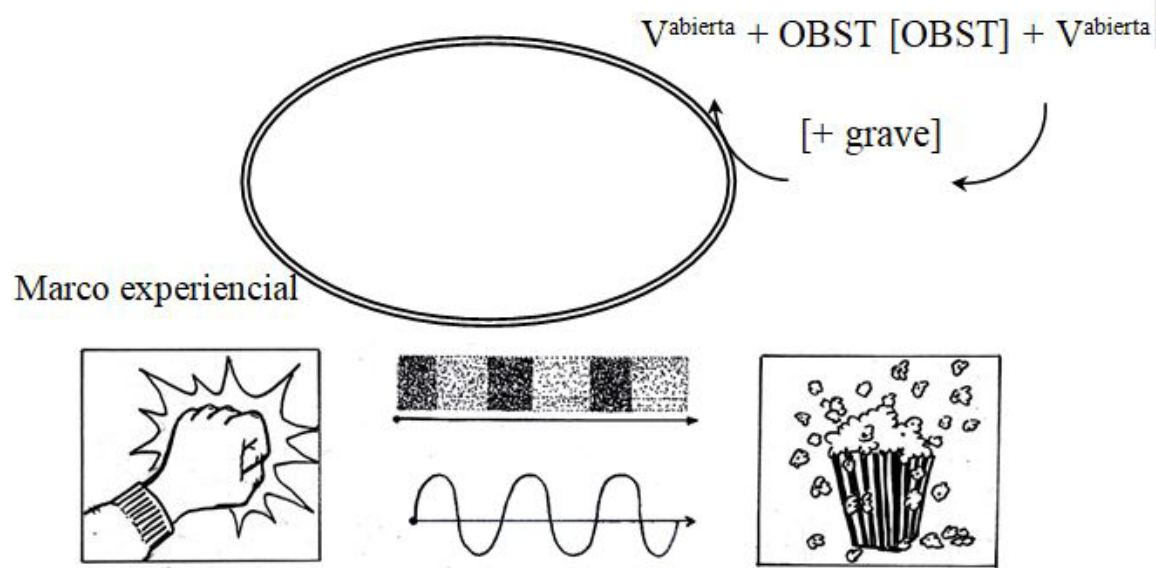

Figura 4. Esquema fonosemántico de las onomatopeyas vinculadas con sonidos graves en el quechua central.

Un onomátopo adicional es el que asocia ambos tipos de sonidos en una sola onomatopeya. Se trata de una sola onomatopeya de este tipo, pero podría establecerse como un caso que ejemplifica la categorización de ambos esquemas en una sola expresión onomatopéyica. Ejemplo es el dato que se corresponde con la emisión del burro. Este se presenta a continuación en (4):

(4) haachin haachin 'el rebuzno del burro'

En el caso propuesto se detecta la secuencia de vocal abierta, que aparece en onomatopeyas vinculadas con sonidos de la realidad que podrían conceptualizarse con el rasgo [+ grave] y la vocal alta anterior que, como vimos, aparece en el onomátopo para sonidos que son [+ agudo]. Este es el siguiente:

$$
\mathrm{V}: \mathrm{ABIERTA}+\mathrm{OBST}+\mathrm{V}^{\mathrm{ANT}}
$$

Otro grupo de datos en los que se evidencia la vocal central baja en estructuras consistentes se asocia con sonidos de la realidad que presentan una indeterminación mayor. Es decir, sonidos con patrones de ruido diferentes en una misma secuencia acústica de la realidad. Los datos son los siguientes: 
(5) chap chap 'sonido que hace el chancho al comer'

lap lap ' 'el sonido que produce el perro al lamer'

sap sap 'el sonido que produce un ave al volar'

par par 'sonido del vuelo de las aves (de pari 'volar')'

kar kar 'ruido de los loros en grupo'

Los tres primeros datos de (5) se engarzan con onomatopeyas que conceptualizan los ruidos generados por la acción de comer o la acción de volar. Estas son bastante imprecisas, a diferencia de los ruidos cortos como el que ocurre cuando se toca la puerta; o el de un claxon, pues este se caracteriza por su continuidad y se percibe como uniforme. En los casos anteriores, el sonido recurrente es la vocal central baja abierta /a/. El onomátopo para sonidos que son acústicamente más imprecisos por implicar diversas fuentes y tiempos de duración es el siguiente:

\section{OBST $T^{\text {CORONAL }}+\mathrm{V}^{\text {CENTRAL-BAJA }}+$ OCL $^{\text {LABIAL }}$ \\ $\mathrm{OBST}^{(\mathrm{LAB})(\mathrm{VEL})}+\mathrm{V}^{\mathrm{CENTRAL-BAJA}}+\mathrm{VIBR}^{\mathrm{CORONAL}}$}

Como se indicó, en los onomátopos anteriores se evidencia un patrón recurrente: la vocal /a/. Sin embargo, esta se posiciona entre un sonido coronal en arranque o sonidos labiales y velares en coda silábica. Además, cuando el arranque es coronal, la coda es labial. Cuando el arranque es labial o velar, la coda es coronal. Ello nos hace suponer lo siguiente: los sonidos labiales y velares forman un patrón con la vocal central baja no redondeada, ya sea en arranque o en coda, de manera que la consonante coronal puede aparecer por defecto en cualquiera de las dos posiciones. Lo importante en estos casos es que se sature la estructura silábica con sonidos que se articulan con un espacio considerable en la cavidad oral. Tal es el caso de los sonidos labiales, velares y la vocal abierta /a/. De acuerdo con lo señalado, el onomátopo podría reducirse a la siguiente posibilidad:

$$
\left[\mathrm{OBST}^{(\mathrm{LAB})(\mathrm{VEL})}\right]+\mathrm{V}^{\mathrm{CENTRAL}-\mathrm{BAJA}}+\mathrm{OCL}\left[{ }^{\mathrm{LABIAL}}\right]
$$

Resulta interesante la forma en que los sonidos que ocurren en posición de arranque y en posición de coda silábica se vinculan, en términos acústicos, con 
los correlatos GRAVE-AGUDO planteados por Jakobson, Fant y Halle (1963). En esta propuesta clásica, se propuso que los sonidos graves son aquellos cuyas articulaciones periféricas son consistentes con una mayor disposición de espacio oral, en contraposición con los sonidos agudos, los cuales generan divisiones en la cavidad oral y, por consiguiente, involucran un espacio menor debido a la división. En otra obra, Jakobson y Halle describen el rasgo de la siguiente manera: “[...] acústicamente - concentración de energía en las frecuencias bajas (vs. altas) del espectro [acústico]; genéticamente - periférico vs. central: fonemas periféricos (velares y labiales) tienen un resonador más amplio y menos dividido que los correspondientes fonemas centrales (palatales y dentales)" (1956, p. 31). Es este patrón el que pareciera reflejar la lengua quechua en la constitución de sus estructuras onomatopéyicas, las cuales han sido descritas de forma preliminar, aunque la ocurrencia de patrones generales podría concebirse en términos más amplios; es decir, se trataría de formas detectables en otras lenguas.

\section{Conclusiones}

a) Las onomatopeyas recolectadas se conforman a través de motivaciones específicas vinculadas con el marco experiencial de los quechuahablantes; a saber, la percepción de los distintos sonidos de la realidad inmediata se conceptualiza a través de onomátopos que, a su vez, están engarzados con significados, de manera que los esquemas fonosimbólicos descritos se constituyen de forma consistente.

b) Los esquemas fonosemánticos suponen un marco de generalización en el que la estructura de los sonidos interactúa con la semántica de la lengua. En tal sentido, la ocurrencia de vocales anteriores relacionadas con sonidos agudos de la realidad y de vocales articuladas con un espacio mayor de la cavidad, cuyas relaciones con sonidos graves son evidentes, se formalizan mediante categorías en las cuales se vinculan lo fonológico y lo semántico.

c) Existen asociaciones de sonido y de significado más paradigmáticas que otras. Así, algunas onomatopeyas reflejan un grado mayor de iconicidad, por ejemplo, los sonidos vinculados con las emisiones de ciertas aves son reflejo de una tendencia que es universal y que ha sido registrada en diversas 
lenguas que carecen de filiación genética. Lo mismo ocurre en el caso de los sonidos asociados con los golpes. Otros datos de tipo más indeterminado conforman patrones menos reconocibles interlingüísticamente, pero que reflejan la motivación por un lado, y la forma en que los quechuahablantes conceptualizan la realidad inmediata a través del lenguaje, pues su función medular es explicitar significados para la lingüística cognitiva.

\section{Agradecimientos}

Este artículo es producto final del curso Seminario de Teoría Lingüistica A (Fonología), dictado por la Dra. Emérita Escobar Zapata en el primer semestre del Doctorado de Lingüística de la Facultad de Letras y Ciencias Humanas de la Universidad Nacional Mayor de San Marcos.

\section{Nota}

1 Aunque el uso de onomatopeyas en otros contextos como el poético podría eventualmente sugerir que, en algún término, los recursos onomatopéyicos pueden ser imitativos (Al-Zubbaidi, 2014, p. 182).

\section{Referencias bibliográficas}

Al-Zubbaidi, H. (2014). The Functions of Onomatopoeia in Modern English and Arabic Poetry: A Study in Selected Poems by Lawrence and al-Sayyab. Advances in Language and Literary Studies, 5 (6), 181-193. https://doi. org/10.7575/aiac.alls.v.5n.6p.181

Boersma, P. \& Weenink, D. (2019). Praat: doing phonetics by computer [Programa de computadora]. Versión 6.0.50. Recuperado en: http://www.praat.org/.

Croft, W. \& Cruse, A. (2004). Cognitive Linguistics Cambridge: Cambridge University Press. https://doi.org/10.1017/CBO9780511803864

De Saussure, F. (1945). Curso de lingüistica general. Buenos Aires: Editorial Losada S. A.

Goldberg, A. E. (1996). Jackendoff and construction-based grammar. Cognitive Linguistics, 7, 3-19. https://doi.org/10.1515/cogl.1996.7.1.3 
Hengeveld, K. (2003). La tipología lingüística. En R. Mairal Usón y J. Gil Fernández (Coords.), En torno a los universales lingüísticos (pp. 89112). Madrid: Akal.

Himmelmann, N. P. (1998). Documentary and descriptive linguistics. Linguistics, 6, 161-195. https://doi.org/10.1515/ling.1998.36.1.161

Hinton,L., Nichols, J.\& Ohala,J.(1994). Sound Symbolism. Nueva York: Cambridge University Press. https://doi.org/10.1017/CBO9780511751806

Ibarretxe A., I. (2012). Análisis lingüístico de las onomatopeyas vascas. Oihenart, 27, 129-177.

Jakobson, R., Fant G. M. \& Halle, M. (1963). Preliminaries to Speech Analysis: The Distinctive Features and their Correlates. Cambridge: The MIT Press.

Jakobson, R. \& Halle, M. (1956). Fundamentals of Language. La Haya: Mouton $\&$ Co.

Jannedy, S., Poletto, R. \& Weldon, T. (1994). Language Files: Materials for an Introduction to Language and Linguistics. Columbus: Ohio State University Press.

Kritikos, E. (2015). Applying Current Methods in Documentary Linguistics in the Documentation of Endangered Languages: A Case Study on Fieldwork in Arvanitic. Athens Journal of Philology, 2 (4), 243-254. https://doi. org/10.30958/ajp.2-4-3

Lakoff, G. (1987). Women, fire and dangerous things: What categories reveal about the mind. Chicago: Chicago University Press. https://doi. org/10.7208/chicago/9780226471013.001.0001

Moreno C., J. C. (2016). Onomatopeya, delocutividad y fonosimbolismo en la ornitonimia latinoamericana. Liburna, 9, 117-166.

Nartey, M. (2013). On conceptualizing documentary linguistics as an independent field of linguistic research: An effective approach to preserving endangered languages. English for Specific Purposes World, 41, 14. 
Nathan, D. (2010). Sound and unsound practices in documentary linguistics: towards an epistemology for audio. Language Documentation and Description, 7, 262-284

Riemann, W. (2010). Basic oral language documentation. Language Documentation \& Conservation, 4, 254-268.

Rocha M., R. (2010). El sufijo "infinitivizador" en quechua. Letras (Lima), 81(116), 153-165. https://doi.org/10.30920/letras.81.116

Rocha M., R. (2011). Interacción entre la fonología y la morfología: disimilación en el quechua de Aurahuá. Fabla, 4, 151-168.

Sadick N., R. \& Nartey, M. (2014). Language Corpora: The Case for Ghanaian English. 3L: The Southeast Asian Journal of English Language Studies, 20 (3), 79-92. https://doi.org/10.17576/3L-2014-2003-07

Sierra P., C. (2010). Quechua onomatopeyankuna. Pucallpa: Curso de Lingüística Aplicada para Lenguas Vernáculas.

Taylor, J. R. (2003). Cognitive Grammar. Oxford: Oxford University Press.

Voronin, S. (2005). Iconocity, Glottogenesis, Semiosis. San Petersburgo: Universidad de San Petersburgo. 